\title{
A COMPUTATIONAL MODEL OF DRIVER DECISION MAKING AT AN INTERSECTION CONTROLLED BY A TRAFFIC LIGHT
}

\author{
Terry Stanard and Robert J. B. Hutton \\ Klein Associates Inc. \\ Fairborn, Ohio USA \\ E-mail: tstanard@decisionmaking.com, rob@decisionmaking.com \\ Walter Warwick, Stacey McIlwaine, and Patricia L. McDermott \\ Micro Analysis and Design \\ Boulder, Colorado USA \\ E-mail: wwarwick@maad.com, smcilwaine@maad.com, pmcdermott@maad.com
}

\begin{abstract}
Summary: An important challenge associated with driving simulation development is the computational representation of agent behaviors. This paper describes the development of a preliminary autonomous agent behavior model (based on the Recognition-Primed Decision (RPD) model, and Hintzman's multiple-trace memory model) mimicking human decision making in approaching an intersection controlled by a traffic light. To populate the model, an initial Cognitive Task Analysis was conducted with six drivers to learn the important cues, expectancies, goals, and courses of action associated with traffic light approach. The agent model learns to associate environmental cues (such as traffic light color) with expectancies of upcoming events (like light color change) and appropriate courses of action (such as decelerating). At present, the model is currently being evaluated for its successful representation of the RecognitionPrimed Decision Making process.
\end{abstract}

\section{INTRODUCTION}

The computational representation of agent behavior is an important challenge associated with driving simulation development. Although agent behavior may be scripted for a specific simulation run, this constrains the level of dynamic interaction a subject may experience within the virtual world. An alternative is the creation of autonomous, intelligent agents within the environmental model. This permits dynamic interactions with the subject, however, the agent behavior may or may not effectively mimic human behavior and thus presents an unrealistic driving environment. To adequately mimic driver behavior, the agent model must be based on both empirical data and a theory of human performance while driving. In this paper, a preliminary computational model of autonomous agent behavior in approaching an intersection controlled by a traffic light is presented, based on an applied theory of decision making known as Recognition-Primed Decision (RPD) (Klein, 1998; Klein, 1989). This computational model, although preliminary, is a step towards the development of more autonomous, human-like agents in a simulation environment.

The paper begins with a description of RPD, a model of the expert's decision-making process. Next, a Cognitive Task Analysis is described. The Cognitive Task Analysis was conducted to populate the key features of a computational recognition-primed decision making model during 
the approach to an intersection controlled by a traffic light. Finally, a computational agent model, which mimics some aspects of a human's behavior when approaching a traffic light, is explained.

\section{RECOGNITION-PRIMED DECISION MAKING}

Recognition-primed decision making is a phenomelogical description of how expert performers make decisions in situations characterized by time constraints, uncertainty, and high stakes. The RPD model originally grew out of the interviews and observations of firefighter commanders confronting real-life fires (Calderwood, Crandall, \& Klein, 1987) and has since been developed and demonstrated in over 50 domains such as driver route planning (Hutton, Thordsen, Militello, \& Heaton, 1995), computer hackers and security experts (McCloskey, Stanard, \& Armstrong, 2001), weather forecasters, and neonatal intensive care nurses (Klein, 1998).

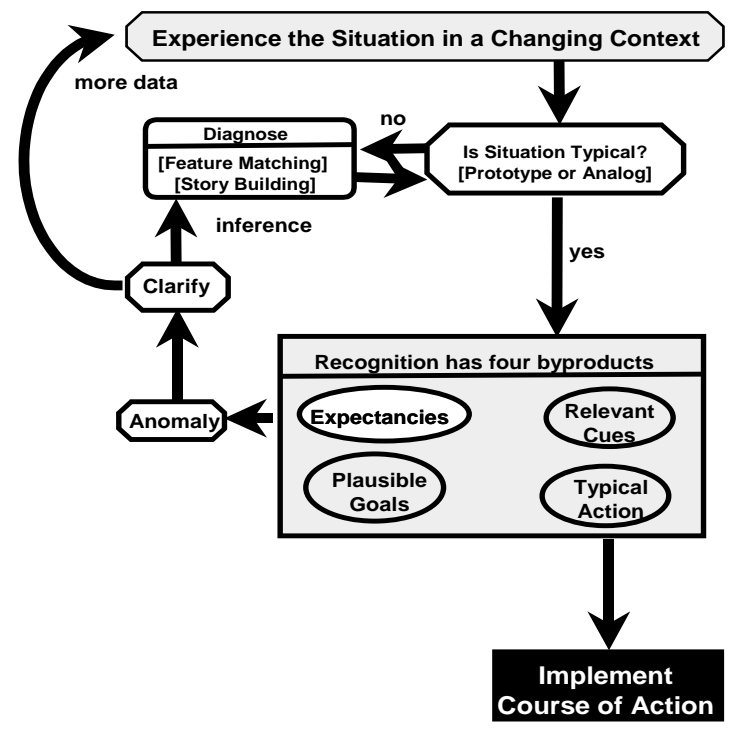

In routine situations (RPD Variation 1: Simple Match), the expert experiences the world in a dynamic context and comes to recognize a situation as typical. Typicality has four components associated with it: relevant cues for that situation, expectancies, plausible goals, and plausible courses of action. Once the situation is recognized as familiar, a single course of action is obvious and is implemented. This basic process contrasts with rational-choice models of decision making, where several action alternatives are generated and the relative merits of each are evaluated before one is selected and executed.

Figure 1. Recognition-Primed Decision (RPD) model, Variation 2.

Figure 1 shows RPD Variation 2: Diagnose the situation. Sometimes, the decision maker engages in more deliberate diagnosis because the situation is not immediately recognized, and information is actively sought to find cues and features that bring to light the nature or type of situation. In other cases, the situation type may initially be recognized, but incorrectly. As events pan out, expectancies of future events are violated and the decision maker seeks clarification of the situation through the diagnostic process. Feature matching and storybuilding are two common processes for diagnosing a situation.

\section{COGNITIVE TASK ANALYSIS}

Variations 1 and 2 of the RPD model were applied towards the development of a preliminary driver decision-making process and computational model. A limited Cognitive Task Analysis study was conducted to populate the elements of the model in the context of an approach to a traffic light. 
Cognitive Task Analysis is a set of qualitative interviewing and observational methods used to uncover the cognitive expertise associated with a complex task. Typically, Cognitive Task Analysis uncovers important information associated with a task, strategies for making sense of data, cognitive challenges, and novice errors. In this study, we conducted individual, one-hour interviews with four drivers having a minimum of two years driving experience ("experts”), and two drivers with less than two years experience ("novices"). The interview focused on general and specific issues surrounding decision making while approaching an intersection controlled by a traffic light, including:

- Prominent cues and environmental factors that the driver attends to

- Expectancies that are formed

- "Red flags" that signal something is amiss

- Goals that the driver may be trying to achieve or balance

- Courses of action that may be followed

Table 1 below lists the major goals, cues, expectancies, and courses of actions that participants reported. One limitation in this format is that cues and expectancies are often not easily expressed verbally, but rather are perceptual quantities difficult to articulate in a few descriptive words. The interview results were then compiled into mappings of the goals, cues, and expectancies onto courses of action. Not all mappings were directly drawn from the interview data-many mappings were created based on research team members' understanding of driving.

An important finding from the driver interviews not described in the table is the "point of no return” (PONR). Some participants reported that there is a point in the approach to the traffic light when a decision has to be made to go or stop. This likely conforms to a minimum safe stopping distance, which is a function of approach speed, road conditions, brake performance, reaction time, etc. If the driver nears the PONR, the decision must be made to stop immediately or go. Crossing the PONR means the driver has no choice but to go (or risk stopping within the intersection).

Table 1

Components of the Decision-making Context at an Intersection Controlled by a Traffic Light

\begin{tabular}{|l|l|l|l|}
\hline \multicolumn{1}{|c|}{ Goals } & \multicolumn{1}{|c|}{ Cues } & \multicolumn{1}{c|}{ Expectancies } & \multicolumn{1}{c|}{ Course of Action } \\
\hline - Don't break the law & - Light Color & Lead car actions: & - Accelerate \\
- Don't get caught & - Distance to light & - Going through light & - Decelerate \\
- Don't hit anything or be & - Auto speed & - Stopping & - Stop (controlled) \\
hit & - Presence of cop & - Not moving & - Stop (hard break) \\
- Minimize drive time & - Pedestrian presence & & - Maintain speed \\
- Preserve momentum & - Presence of lead car & Light: & - Change lanes \\
- Maintain traffic flow & - Presence of cross traffic & -Going to change & \\
- Avoid sudden stops & -Not going to change & \\
& Other vehicle actions: & -Can't make it before it changes & \\
& - Slowing lead & - Pedestrian moving & \\
& - Turning on-coming & & \\
& - Turning cross traffic & &
\end{tabular}




\section{COMPUTATIONAL MODEL}

A real-world driving environment encompasses a variety of subtle interactions between the driver and a dynamic situation. To facilitate the initial development of a working computational model, we decided to simplify the decision-making context. Selected cues, expectancies, and courses of action from Table 1 were included. Goals were not considered for the initial model, and some environmental factors such as pedestrians, lead vehicles, and turning traffic were not featured. One course of action (lane change) was also eliminated for the initial model development.

\section{Memory and Typicality}

Recognition-primed decision making is an abstract, qualitative model, and the transformation to a computational model presents a variety of challenges. It does not address all the cognitive structures supporting the decision-making process diagrammed in Figure 1. For instance, recognition-primed decision making assumes an experience base that the decision maker relies upon, but the model does not describe the Long Term Memory (LTM) store. It also posits a decision maker who recognizes a situation as typical, but the model does not describe how this typicality is formed from memory. These two issues of LTM and typicality are addressed using Hintzman's multiple-trace memory model (Hintzman, 1986). Within the multiple-trace model, each experience of the agent is encoded as a unique trace in LTM. A multiple-trace memory is a collection of all the experience traces, rather than a store of experience types. Recognition is a process of comparing a given situation to each trace in memory, and producing a similarity value for the trace. The similarity values of all trace comparisons are combined and formed into an "echo" representing the typicality of the situation.

\section{Decision Cycle}

Figure 2 and labels A-D show how a single cycle of the computational driver model works in relation to the RPD model. The driver's memory is a store of multiple experiences at the same intersection. During an episode, the driver approaches the intersection and initially samples the environment (A). The sampling strategy is determined probabilistically and includes cues such as traffic light color (green, yellow, red), relative distance to the light (PONR, Not PONR), crosstraffic presence (yes, no), and law enforcement presence (yes, no). This constitutes the first phase

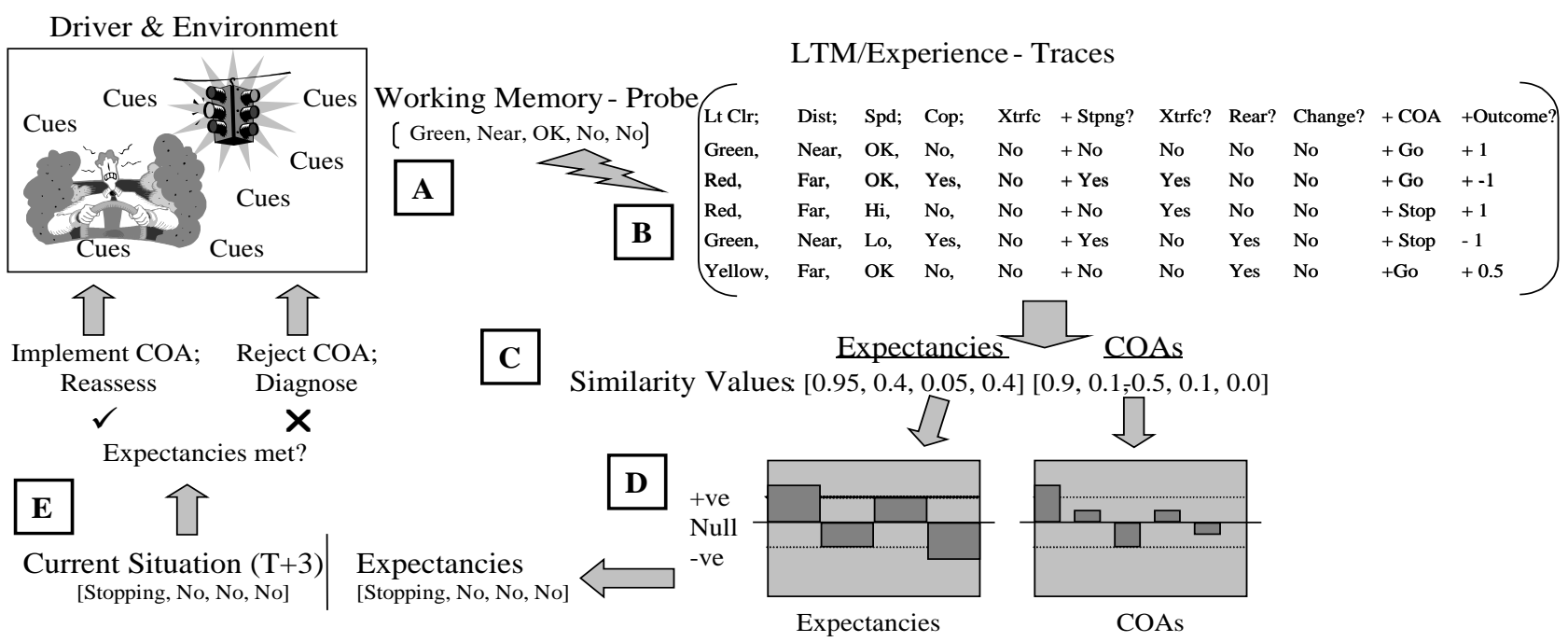

Figure 2. Computational model of recognition-primed decision making at a traffic light intersection. 
of RPD: "Experience the environment in changing context." The environment sample is stored in short-term memory (STM) and used to "probe" LTM (B). Probing LTM consists of computing a similarity value between the contents of STM and each trace in LTM. These similarity values are used, in turn, to produce an echo that represents the weighted contribution of each trace in LTM to the components of typicality. One of these components is a set of four dichotomous expectancies (car is stopping, cross traffic is present, rear vehicle will be present, and light will change). Positive values indicate "yes," negative values indicate "no" (C). The echo also indicates which of five different courses of action (go, stop, accelerate, decelerate, maintain speed) has been “recognized.” Recognition in this context includes some "off-line” statistical analysis to determine whether a single course of action has in fact been recognized and whether that course of action has been successful in past similar situations. The comparative process of probing LTM and returning an echo constitutes recognition in this model: "Is situation typical?" What are recognized expectancies for a given situation, and appropriate (or inappropriate) courses of action?

At this point, the driver takes another sample of the current environment to confirm or invalidate the expectancies (E). If expectancies are met, the course of action is implemented and the results of that action are recorded; if the expectancies are violated, the driver reassesses the situation starting with a fresh set of cues from the environment (A) and another pass through the probeecho cycle. This constitutes "anomaly” and "clarify” in the RPD model (Klein, 1989). Once a course of action has been implemented (perhaps after several attempts at diagnosis), the results of that action are used to compute a measure of successfulness. The success measure is stored as a new trace in LTM, along with the cue values that prompted recognition and the cue values that were used to evaluate the expectancies.

\section{TEST BED ENVIRONMENT}

The agent model was created using Micro Saint, enhanced with a Visual Basic back end to implement LTM and the recognition and diagnosis routines. At present, the agent model is undergoing qualitative and quantitative tests to evaluate how successfully it mimics human decision-making behavior at an intersection, both in terms of decision-making product and process.

\section{ACKNOWLEDGEMENTS}

We would like to thank Scott Baldwin of the Transportation Research Center, Inc. for his editorial comments on this paper. This work is supported by Dr. Denise Lyons at the Naval Air Warfare Center, Training Systems Division (under contract \#N61339-99-C-0103) and by Dr. Wendy Martinez at the Office of Naval Research (under contract \#N00014-00-M-0069). We are grateful for their input and encouragement. 


\section{REFERENCES}

Calderwood, R., Crandall, B. W., \& Klein, G. A. (1987). Expert and novice fireground command decisions (Final Report under contract MDA903-85-C-0327 for the U.S. Army Research Institute, Alexandria, VA). Fairborn, OH: Klein Associates Inc.

Hintzman, D. L. (1986). "Schema abstraction" in a multiple-trace memory model. Psychological Review, 93(4), 411-428.

Hutton, R. J. B., Thordsen, M., Militello, L., \& Heaton, J. (1995). Cognitive task analysis of drivers and traffic management personnel: Implications for human factors guidelines for advanced traveler information systems (Contract FHWA DTFH61-95-C-00017 for COMSIS Corporation). Fairborn, OH: Klein Associates Inc.

Press.

Klein, G. (1998). Sources of power: How people make decisions. Cambridge, MA: MIT

Klein, G. A. (1989). Recognition-primed decisions. In W. B. Rouse (Ed.), Advances in man-machine systems research (Vol. 5, pp. 47-92). Greenwich, CT: JAI Press, Inc.

McCloskey, M. J., Stanard, T. W., \& Armstrong, A. A. (2001). Cognitive analysis of the information security domain (Technical AFRL-HE-WP-TR-2001-0041 for Air Force Research Laboratory, Wright-Patterson AFB, OH). Fairborn, OH: Klein Associates Inc. 